\title{
EFECTOS DE CONSEGUIR LA PRIMERA POSESIÓN DEL BALÓN SOBRE EL MARCADOR EN LOS CAMPEONATOS DEL MUNDO DE WATERPOLO 2003 Y 2007
}

\author{
DR. FRANCISCO MANUEL ARGUDO ITURRIAGA \\ Doctor en Psicología por la Universidad de Valencia (España) \\ Profesor del Departamento de Educación Física, Deporte y Motricidad Humana de la Universidad \\ Autónoma de Madrid (Cantoblanco - Madrid - España) \\ E-mail: quico.argudo@uam.es

\section{DR. JOSÉ LUIS ARIAS ESTERO} \\ Doctor en Ciencias de la Actividad Física y del Deporte por la Universidad Autónoma de Madrid \\ (España), Profesor del Departamento de Ciencias de la Actividad Física y del Deporte de la \\ Universidad Católica San Antonio de Murcia (Guadalupe - Murcia - España) \\ E-mail: jlae84@hotmail.com, jlarias@pdi.ucam.edu
}

\section{DRA. ENCARNACIÓN RUIZ LARA}

Doctora en Ciencias de la Actividad Física y del Deporte por la Universidad de León (España), Profesora del Departamento de Ciencias de la Actividad Física y del Deporte de la Universidad Católica San Antonio de Murcia (Guadalupe - Murcia - España)

E-mail: erlara@pdi.ucam.edu

\section{RESUMEN}

El objetivo de este estudio fue analizar la influencia de tener la primera posesión del balón sobre el marcador parcial y final en waterpolo. Se grabaron los 192 partidos disputados por todos los equipos participantes en los Campeonatos del Mundo de 2003 y 2007. La muestra estuvo formada por todos los equipos participantes en ambas competiciones. Se utilizó la metodología observacional. Se filmaron los partidos y posteriormente fueron analizados con el software Polo análisis v. 1.0 directo. El 43,4\% de los equipos masculinos y el 52,6\% de los equipos femeninos que consiguieron la primera posesión del balón disfrutaron de un resultado parcial favorable a lo largo de los periodos. Los equipos masculinos que ganaron más del 50\% de los partidos cogieron el primer balón en más de un periodo. Esto ocurrió en la categoría femenina en más de dos periodos. En conclusión, coger el primer balón en un mayor número de periodos se relaciona con un marcador parcial y final favorable.

PALABRAS CLAVE: Waterpolo; rendimiento; primera posesión; análisis de juego. 


\section{INTRODUCCIÓN}

En la última década han aumentado los estudios preocupados por los factores que determinan el rendimiento en waterpolo (FELTNER; TAYLOR, 1997; HATZIGEORGIADIS, THEODORAKIS; ZOURBANOS, 2004; KAVOURAS et al., 2006; KONSTANTAKI; TROWBRIDGE; SWAINE, I998; MARLOW et al., 1998; PLATANOU, 2004; SANDERS, 1999a, 1999b; SMITH, 1998; SMITH, 2004; STEEL et al., 2007; TSEKOURAS et al., 2005). Actualmente existe un mayor interés por el análisis de los factores técnico-tácticos (ARGUDO et al., 2007a, 2007b; ARGUDO; RUIZ; ALONSO, 2008). El análisis técnico-táctico en los deportes de equipo normalmente demanda la estructuración de unidades tan pequeñas como exija el objeto de estudio. Estas unidades de análisis se suelen corresponder con las posesiones de balón (ARGUDO; LLORET, 2007). Estas permiten tener indicadores complementarios para conocer el rendimiento de los equipos (HUGHES; BARTLETT, 2002; LAGO; MARTÍN, 2007).

A diferencia que en otros deportes, en waterpolo el estudio de las posesiones de balón se ha realizado, principalmente, como soporte para la explicación de las acciones de juego (ARGUDO et al., 2007a, b). Se han dejado de lado, hasta ahora, aspectos considerados importantes sobre el resultado final en otros deportes, como la influencia del número de posesiones (JIMÉNEZ; RUIZ, 2006; RAMPININI et al., 2007) y la duración de dichas posesiones (JAMES; JONES; MELLALIEU, 2004; MONTANER; MONTANER, 2004). Uno de los momentos más intensos de los partidos de waterpolo es el de la obtención de la primera posesión del balón al principio de cada periodo de juego. Esta circunstancia conlleva un desgaste inicial de los jugadores para que su equipo obtenga el primer balón.

Recientemente, se han publicado varios estudios que analizaron la influencia de coger el primer balón sobre el marcador parcial y final (ARGUDO, 20 I0; ARGUDO; ARIAS; RUIZ, 2009, 20 l 0, 20 l la, 20 l l b; ARGUDO et al., 201 I; ARIAS, 20 I0). Estos estudios parecen no aportar datos estables sobre el efecto de este indicador en el resultado de los partidos de waterpolo. Por este motivo, parecen necesarios nuevos estudios para intentar encontrar un patrón de comportamiento estable de los resultados.

El objetivo de este estudio fue analizar la influencia de coger el primer balón sobre el marcador parcial y final en waterpolo masculino y femenino. Específicamente, se analizó si coger el primer balón en un mayor número de periodos se relaciona con un marcador parcial y final favorable y en qué periodos se produce la relación con un marcador parcial favorable. 


\section{PARTICIPANTES}

La población objeto de estudio estuvo compuesta por los equipos de categoría masculina y femenina participantes en los Campeonatos del Mundo celebrados en Barcelona-2003 y Melbourne-2007. Los partidos analizados fueron todos los disputados a lo largo de los campeonatos, lo que supuso un total de 192. Al tratarse de dos campeonatos del Mundo, a los equipos se les supuso un nivel elevado y homogéneo de competencia. La muestra estuvo formada por todos los equipos participantes en ambas competiciones.

\section{DISEÑO}

Se utilizó la metodología observacional (ANGUERA; BLANCO, 2003), para analizar qué equipo conseguía la primera posesión del balón en cada uno de los cuatro periodos de cada partido y si este hecho guardaba relación con el resultado ganador, perdedor o empate. La unidad de análisis fue la primera posesión de balón de cada uno de los periodos. Las variables y las categorías objeto de estudio fueron:

I. Periodo en el que se disputaba la primera posesión. Se categorizó en: a) primer periodo, b) segundo periodo, c) tercer periodo y d) cuarto periodo.

2. Equipo que obtuvo la primera posesión. Se categorizó atendiendo a cada uno de los doce equipos participantes, de manera que se establecieron doce categorías.

3. Resultado del periodo. Se categorizó en: a) equipo ganador, b) equipo perdedor y c) empate.

4 Resultado final del partido. Se categorizó en: a) equipo ganador, b) equipo perdedor y c) empate.

MATERIAL

El material empleado para la realización de este trabajo fue de diferente naturaleza: a) para la filmación de partidos y b) para la recogida, almacenamiento y tratamiento de los datos:

I. Una cámara de vídeo digital (JVC, GR-DVL 9000 EG) y un trípode.

2. Instrumento de registro: Polo análisis V. I .0 directo (ARGUDO; ALONSO; FUENTES, 2005). 
3. Instrumento de observación: sistema de categorías.

4. Paquete estadístico SPSS 15.0. para Windows.

\section{PROCEDIMIENTO}

Se obtuvo la autorización de la Federación Internacional de Natación para filmar los partidos de los dos campeonatos del mundo y el visto bueno del Comité de Ética de la Universidad del primer autor. Se filmaron todos los partidos de competición correspondientes a los campeonatos del Mundo de 2003 y 2007. La técnica de filmación partió del enfoque inicial al centro del espacio de juego, para posteriormente abrir el zoom y captar los límites del espacio en cuestión. Se adiestró a dos sujetos (ANGUERA, 2003), con una experiencia de más de 300 horas en observación y registro de aspectos relacionados con el desarrollo de los partidos de waterpolo. No obstante, se obtuvo la fiabilidad intraobservador, dejando un periodo de no observación de siete días, desde la primera observación hasta la segunda. La fiabilidad alcanzada, mediante el coeficiente Kappa, de fue superior al 95. Por último, se procedió a la toma de datos mediante un registro sistemático. Para lo cual se utilizó el software Polo análisis v. I.0 directo (ARGUDO; ALONSO; FUENTES, 2005). Este es un instrumento desarrollado para la evaluación táctica cuantitativa en waterpolo a tiempo real. La observación fue sistemática y se realizó a posteriori, sobre los vídeos grabados. El registro de los criterios observados se realizó según las instrucciones del software Polo análisis v. I .0 directo (ARGUDO; ALONSO; FUENTES, 2005).

\section{ANÁLISIS ESTADÍSTICO}

Los datos registrados mediante el programa Polo análisis v. I .0 directo se exportaron para archivarlos a través del paquete estadístico SPSS 15.0. para Windows, con el fin de ser tratados estadísticamente. Se empleó la prueba de Chi-Cuadrado para conocer la influencia de la obtención del primer balón sobre el marcador parcial y el marcador final de cada periodo. También se utilizó el test de asociación mediante el estadístico Gamma, para analizar si existía influencia entre el número total de primeras posesiones conseguidas y el resultado final, en tanto en cuanto, ganar, perder o empatar. Todos los análisis fueron acompañados de sus respectivas tablas de contingencia. 


\section{RESULTADOS}

Para la categoría masculina se encontraron diferencias estadísticamente significativas en la influencia de coger el primer balón sobre el marcador parcial $\left(\chi^{2}=\right.$ 5,304; g.l. = 2; $p=$,05). Como muestra la Tabla I, en el 43,4\% de las ocasiones que se consiguió la primera posesión del balón se terminó ganando el periodo. Para la categoría femenina se encontraron diferencias estadísticamente significativas en la influencia de coger el primer balón sobre el marcador parcial $\left(\chi^{2}=40,625\right.$; g.l. $=$ $2 ; p=, 000)$. En el $52,6 \%$ de las ocasiones que se consiguió la primera posesión del balón se terminó ganando el periodo.

Tabla I - Influencia de la primera posesión de balón sobre el marcador parcial

\begin{tabular}{lcccc}
\hline \multicolumn{1}{c}{ Primer balón } & & Gana & Pierde & Empata \\
\hline Categoría masculina & & & & \\
Coge & $n$ & 167 & 138 & 78 \\
No coge & $\%$ & 43,4 & 36 & 20,3 \\
Categoría femenina & $\%$ & 138 & 167 & 78 \\
Coge & $n$ & 36 & 43,4 & 20,3 \\
No coge & $\%$ & 202 & 121 & 61 \\
& $n$ & 52,6 & 31,5 & 15,9 \\
\hline
\end{tabular}

Para la categoría masculina no se encontraron diferencias estadísticamente significativas en la influencia de coger el primer balón sobre el marcador parcial en los periodos I $\left(\chi^{2}=, 4 \mid 6 ;\right.$ g.l. $\left.=2 ; p=, 8 \mid 2\right), 2\left(\chi^{2}=, 247 ;\right.$ g.l. $\left.=2 ; p=, 884\right) y$ $4\left(\chi^{2}=1,926 ;\right.$ g.l. $\left.=2 ; p=, 382\right)$. Por el contrario, en el periodo 3 las diferencias fueron significativas $\left(\chi^{2}=8,9 \mid 9 ;\right.$ g.l. $\left.=2 ; p=, 0 \mid 2\right)$. Como muestra la Tabla 2, el porcentaje de equipos que cogieron el primer balón y ganaron fue mayor que el porcentaje de aquellos que no cogieron el primer balón y ganaron y que los que cogieron el primer balón y perdieron en los periodos primero, tercero y cuarto. Las diferencias fueron menores en los periodos primero y cuarto. En el segundo periodo ocurrió al contrario. Para la categoría femenina se encontraron diferencias estadísticamente significativas en la influencia de coger el primer balón sobre el marcador parcial en los periodos I $\left(\chi^{2}=14,107 ; \mathrm{g} . \mathrm{l} .=2 ; \mathrm{p}=, 001\right), 2\left(\chi^{2}=16,122\right.$; g.l. $=2 ; p=, 000)$ y $4\left(\chi^{2}=8,308 ;\right.$ g.l. $\left.=2 ; p=, 016\right)$. Por el contrario, en el periodo 3 las diferencias no fueron significativas $\left(\chi^{2}=4,278 ;\right.$ g.l. $=2 ; p=, \mid$ । 8 ). El porcentaje de equipos que cogieron el primer balón y ganaron fue mayor que el porcentaje de aquellos que no cogieron el primer balón y ganaron y que los que cogieron el primer balón y perdieron en todos los periodos. Las diferencias fueron mayores en los periodos iniciales en comparación con los finales. 
Tabla 2 - Influencia de la primera posesión de balón sobre el marcador parcial por periodos

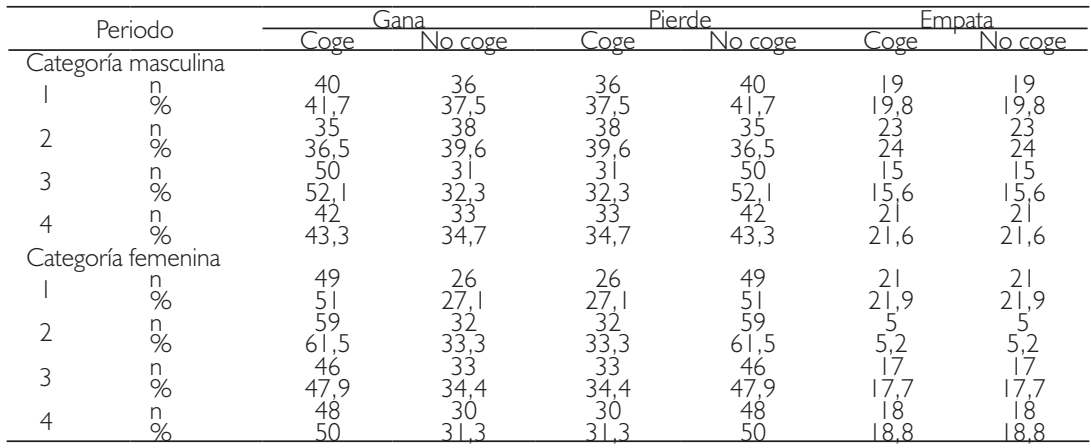

Para la categoría masculina se encontró una asociación significativa entre el número de periodos iniciados con posesión de balón con respecto al marcador final $(\gamma=-0,236$; e.t. $=0,098, p=, 017)$. Como muestra la Tabla 3, los equipos que cogieron el balón en dos, tres y cuatro periodos ganaron más del $50 \%$ de los partidos. Para la categoría femenina se encontró una asociación significativa entre el número de periodos iniciados con posesión de balón con respecto al marcador final $(\gamma=$ $-0,468$; e.t. $=0,082, p=, 000)$. Los equipos que consiguieron la primera posesión en tres $(61,9 \%)$ y cuatro periodos $(77,4 \%)$ ganaron los partidos. Los equipos que consiguieron la posesión de balón en dos periodos y no la consiguieron en otros dos tuvieron el $50 \%$ de posibilidades de ganar o perder el partido.

Tabla 3 - Influencia del número de periodos iniciados con posesión de balón respecto al marcador final

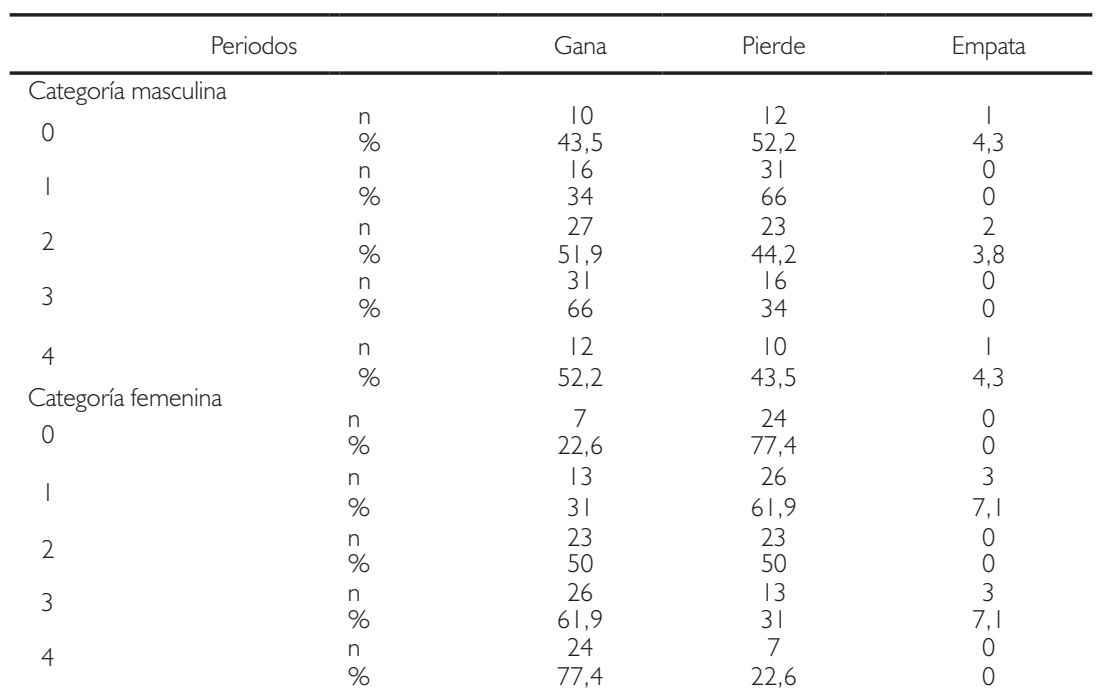




\section{DISCUSIÓN}

El objetivo de este estudio fue analizar la influencia de coger el primer balón sobre el marcador parcial y final en waterpolo masculino y femenino. Específicamente, se analizó si coger el primer balón en un mayor número de periodos se relaciona con un marcador parcial y final favorable y en qué periodos se produce la relación con un marcador parcial favorable. El 43,4\% de los equipos masculinos y el $52,6 \%$ de los equipos femeninos que consiguieron la primera posesión del balón disfrutaron de un resultado parcial favorable a lo largo de los periodos en cuestión. Los equipos masculinos que consiguieron la posesión del balón en el tercer periodo $(52,1 \%)$ alcanzaron un resultado significativamente favorable. En los periodos iniciales y el final la consecución de la primera posesión se relacionó con unos porcentajes favorables en cuanto al resultado positivo, aunque no significativamente. Sin embargo, los equipos femeninos que consiguieron la posesión del balón en los periodos primero (5 I \%), segundo (61,5\%) y cuarto (50\%) alcanzaron un resultado significativamente favorable. Estos datos contrastan con la idea de que algunos entrenadores centran la importancia de los partidos al principio (SAMPAIO, 200I; SAMPAIO et al., 2006), durante el último periodo (KAMINSKY, I 990; SAMPAIO; LORENZO; RIBEIRO, 2006), o durante los instantes iniciales y finales (NEWELL; KNIGHT, 1986; SAMPAIO; LORENZO; RIBEIRO, 2006).

El patrón de los resultados fue similar entre ambas categorías al comparar el efecto sobre el marcador parcial, pero diferente al compararlo distinguiendo cada periodo. En el caso de las mujeres las diferencias entre los equipos que consiguieron el primer balón y ganaron con respecto a los equipos que no lo cogieron y perdieron fue mayor en comparación con los hombres. Además, en los equipos femeninos se observó que en tres periodos la relación con coger el primer balón fue significativa. Esto sugiere que en waterpolo femenino las diferencias entre los equipos son mayores que en la categoría masculina (ARGUDO; RUIZ, 2006). Las relaciones significativas se produjeron en la categoría masculina en el tercer periodo y en la femenina en los dos periodos iniciales y el final. Partiendo de la base de que las diferencias fueron mayores en los equipos femeninos, parece que estos centraron sus esfuerzos para ganar los partidos en los momentos iniciales.

Los equipos masculinos que ganaron más del $50 \%$ de los partidos cogieron el primer balón en más de un periodo. Los resultados del presente estudio con respecto a la categoría masculina sólo coinciden con los de Argudo et al. (20 I l), y Argudo (20 I0). En el presente estudio las diferencias fueron significativas esta- 
dísticamente a diferencia que en los estudios de Argudo, Arias e Ruiz (20 l la), Argudo, Arias e Ruiz (20 I l b), Argudo, Arias e Ruiz (20 l 0), y Arias (20 I 0). Esto ocurrió en la categoría femenina en más de dos periodos. Estos resultados siguieron el mismo patrón observado en los estudios que analizan a equipos femeninos (ARGUDO; ARIAS; RUIZ, 2009, 20 I0, 20। la, 20l l b; ARGUDO et al., 20II; ARIAS, 20I0).

A medida que disminuyó el número de periodos en que los equipos obtuvieron la primera posesión también disminuyó el porcentaje de victorias. Este resultado parece seguir la línea de que lo que ocurre en ciertos momentos de los partidos repercute sobre el resultado final (HUGHES et al., I 998; MCGARRY et al., 2002). La diferencia entre las categorías residió en que la masculina pudo ganar más de la mitad de los partidos cogiendo el balón en dos o más periodos, mientras que la femenina necesitó tres o más. Los resultados del presente estudio con respecto a la categoría masculina coinciden con los obtenidos por Argudo, Arias e Ruiz (20 I l b) y Arias (20 I 0). Sin embargo, no coincidieron con el resto de estudios al respecto (ARGUDO, 20I0; ARGUDO; ARIAS; RUIZ, 2009, 20 I0, 20 I la; ARGUDO et al., 20 I I). Los resultados del presente estudio con respecto a la categoría femenina coinciden con los obtenidos por Argudo et al. (20 I l), Argudo, Arias e Ruiz (20 I l b), y Argudo, Arias e Ruiz (20 I 0). Sin embargo, no coincidieron con el resto de estudios al respecto (ARGUDO, 20 I0; ARGUDO; ARIAS; RUIZ, 2009, 20I Ia; ARIAS, 20I0).

Estos resultados deben ser analizados con precaución al no existir un patrón estable en los resultados, aunque para la categoría femenina los resultados parecen más estables. Son necesarias nuevas investigaciones que permitan establecer patrones de comportamiento al respecto. A pesar de esto, los resultados presentados tienen una transmisión directa a la práctica real, debido a que el análisis del desarrollo de las posesiones de balón permite obtener indicadores de rendimiento necesarios a la hora de estructurar la preparación de los equipos (HUGHES; BARTLETT, 2002; LAGO; MARTÍN, 2007).

\section{CONCLUSIÓN}

En función del análisis realizado en este estudio se obtienen las siguientes conclusiones fundamentales: a) coger el primer balón en un mayor número de periodos se relaciona con un marcador parcial y final favorable, b) para la categoría masculina coger el primer balón en el tercer periodo se relaciona con un marcador parcial favorable en ese periodo y c) para la categoría femenina coger el primer 
balón en los dos periodos iniciales y en el final se relaciona con un marcador parcial favorable en esos periodos.

\section{Efeitos de conseguir a primeira posse da bola sobre o marcador nos campeonatos do mundo de polo aquático 2003 e 2007}

RESUMO: O objetivo deste estudo foi analisar a influência de conseguir a primeira posse da bola sobre o marcador parcial e final em polo aquático. Gravaram-se as 192 partidas disputadas por todas as equipes participantes nos Campeonatos do Mundo, de 2003 e 2007. A amostra do estudo foram as equipes participantes das referidas competições. Utilizou-se a metodologia observacional. Os jogos foram filmados e, posteriormente, analisados com o software "Polo Análisis v. 1 .0 directo". O resultado demonstrou que 43,4\% das equipes masculinas e 52,6\% das equipes femininas que conseguiram a primeira posse da bola, desfrutaram de um resultado parcial favorável ao longo dos períodos. As equipes masculinas que ganharam mais de $50 \%$ das partidas, conseguiram a primeira bola em mais de um período. Isto ocorreu na categoria feminina em mais de dois períodos. Em conclusão, conseguir a primeira posse da bola em um maior número de períodos está relacionado com um resultado parcial e final favorável.

PALAVRAS-CHAVE: Polo aquático; rendimento; primeira posse; análise do jogo.

\section{Effect of taking the first ball possession on score during the 2003 and 2007 Water polo World Championships}

ABSTRACT: The purpose of this study was to analyze the influence of taking the first ball possession on the partial and final score in water polo. The 192 matches disputed by team's participants in the 2003 and 2007 Water Polo World Championship were recorded. The sample included all participants on both competitions. The observational methodology was applied. Matches were filmed and later they were analyzed with "Polo Análisis v. 1.0 directo" software. The $43.4 \%$ of men's teams and $52.6 \%$ of the female teams, who got the first ball possession, got a favourable partial result along the periods. The men's teams that won more than $50 \%$ of the matches took the first ball in more than one period. This occurred in the female category in more than two periods. In conclusion, to take the first ball in the majority number of periods is related to favourable partial and final score.

KEY WORDS: Water polo; performance; first possession; game analysis. 


\section{REFERENCIAS}

ANGUERA, M. T. La observación. In: MORENO, C. (Ed.). Evaluación psicológica: concepto, proceso y aplicación en las áreas del desarrollo y de la inteligencia. Madrid: Sanz y Torres, 2003. p. $27 \mid-308$.

ANGUERA, M. T.; BLANCO, A. Registro y codificación en el comportamiento deportivo. In: HERNÁNDEZ, A. Psicología del deporte: metodología. Buenos Aires: Efdeportes, 2003. p. 6-34.

ARGUDO, F. M. Influencia de la primera posesión sobre el marcador parcial y final en el Campeonato del Mundo de Waterpolo 2003. Retos, Murcia, n. 17, p. 86-89, junio 2010.

; LLORET, M. Investigación en waterpolo (1990-2003). Murcia: Diego Marín,

2007.

; ALONSO, J. I.; FUENTES, F. Computerized registration for tactical quantitative evaluation in water polo: polo partido vI.0. In: INTERNATIONAL SYMPOSIUM COMPUTER SCIENCE IN SPORT, 5., 2005, Croatia. Proceedings... Croatia: ISCSS, 2005. v. I, p. I - 18 .

ARGUDO, F. M.; ARIAS, J. L.; RUIZ, E. Efectos de tener la primera posesión del balón sobre el marcador en waterpolo. Revista Internacional de Medicina y Ciencias de la Actividad Física y del Deporte, Madrid, v. II, n. 44, p. 738-748, diciembre 201 I a.

ARGUDO, F. M.; ARIAS, J. L.; RUIZ, E. Efectos de tener la primera posesión del balón sobre el marcador parcial y final en los campeonatos del mundo de Waterpolo de 2005 y 2007. Apunts, Barcelona, n. 106, p. 54-6I, diciembre 201 lb.

ARGUDO, F. M.; ARIAS, J. L.; RUIZ, E.; ALONSO, J. I. Effect of winning the first ball possession on the partial and final score during the 2003, 2005 and 2007 water polo world championships. Perceptual and Motor Skills, Missoula, v. II2, n. 2, p. 349-352, abril 20 I I.

ARGUDO, F. M.; ARIAS, J. L.; RUIZ, E. Influencia de coger el primer balón sobre el marcador parcial y final durante el Campeonato de Europa de Waterpolo masculino de 2006. Kronos, Madrid, v. 8, n. 15, p. 131-138, enero 2009.

ARGUDO, F. M.; ARIAS, J. L.; RUIZ, E. Influencia de coger el primer balón sobre el marcador parcial y final durante el Campeonato del Mundo de Waterpolo de 2005. Cultura, Ciencia y Deporte, Murcia, v. 5, n. 15, p. 181-187, diciembre 2010.

ARGUDO, F. M. et al. Influence of the efficacy values in counterattack and defensive adjustment on the condition of winner and loser in male and female water polo. International Journal of Performance Analysis in Sport, Cardiff, v. 7, n. 2, p. 8I-91, july 2007a.

et al. Diferencias de los valores de eficacia en igualdad numérica entre equipos perdedores en waterpolo masculino y femenino. Kronos, Madrid, v. 6, n. I I, p. 4- |3, jul. 2007b. 
ARGUDO, F. M.; RUIZ, E. Validation of a tactical evaluation process in water polo. In: WORLD CONGRESS ON PERFORMANCE ANALYSIS OF SPORT, 7., 2006, Hungary. Proceedings... Hungary: WCPAS, 2006. v. I, p. 32 - 42.

; ALONSO, J. I. Influence of the efficacy values in numerical equality on the condition of winner or loser in the 2003 Water Polo World Championship. International Journal of Performance Analysis in Sport, Cardiff, v. 8, n. I, p. I 01 - I I 2, jan. 2008.

ARIAS, J. L. Influencia de coger el primer balón sobre el marcador durante el campeonato del mundo de waterpolo de 2007. e-balonmano.com: Revista Digital, Extremadura, v. 6, n. 2, p. 91 - 100, octubre 2010.

FELTNER, M. E.; TAYLOR, G. Three-dimensional kinetics of the shoulder, elbow, and wrist during a penalty throw in water polo. Journal of Applied Biomechanics, Champaign, v. I3, n. 3, p. 347-372, july 1997.

HATZIGEORGIADIS, A.; THEODORAKIS, Y.; ZOURBANOS, N. Self-talk in the swimming pool: The effects of self-talk on thought content and performance on water-polo tasks. Journal of Applied Sport Psychology, v. I6, n. 2, p. 138- I 50, apr. 2004.

HUGHES, M.; BARTLETT, R. M. The use of performance indicators in performance analysis. Journal of Sports Sciences, London, v. 20, n. 10, p. 739-754, oct. 2002.

HUGHES, M.; et al. The perturbation effect and goal opportunities in soccer. Journal of Sports Sciences, London, v. 16, n. I, p. 20, january 1998.

JAMES, N.; JONES, P. D.; MELLALIEU, S. D. Possession as a performance indicator in soccer. International Journal of Performance Analysis in Sport, Cardiff, v. 4, n. I, p. 98- I02, jan. 2004.

JIMÉNEZ, A. C.; RUIZ, L. M. Análisis de las tomas de decisiones en la fase de ataque de las jugadoras aleros de baloncesto. Revista Internacional de Ciencias del Deporte, Madrid, v. 2, n. 4, p. 26-46, oct. 2006.

KAMINSKY, J. Critical game time periods in relation to teams success in collage basketball. 1990. Thesis (Master) - Kent State University, Kent, 1990.

KAVOURAS, S. A. et al. Water polo is associated with an apparent redistribution of bone mass and density from the lower to the upper limbs. European Journal of Applied Physiology, Leeds, v. 97, n. 3, p. 316-321, feb. 2006.

KONSTANTAKI, M.; TROWBRIDGE, E. A.; SWAINE, I. L. The relationship between blood lactate and heart rate responses to swim bench exercise and women's competitive water polo. Journal of Sports Sciences, London, v. 16, n. 3, p. 25I-256, mar. 1998.

LAGO, C.; MARTÍN, R. Determinants of possession of the ball in soccer. Journal of Sports Sciences, London, v. 25, n. 9, p. 969-974, sept. 2007. 
MARLOW, C.; et al. The use of a single case design to investigate the effect of a pre-performance routine on the water polo penalty shot. Journal of Science and Medicine in Sport, Mitchell, v. I, n. 3, p. 143-155, may 1998.

MCGARRY, T.; et al. Sport competition as a dynamical self-organizing system. Journal of Sports Sciences, London, v. 20, n. 10, p. 771-781, oct. 2002.

MONTANER, C.; MONTANER, A. M. Estudio comparativo del tiempo de posesión y sus efectos en el juego entre un equipo masculino y uno femenino de baloncesto de élite. Revista Digita Rendimiento Deportivo, León, n. 8, july 2004. Disponível em: < http://www.rendimientodeportivo.com/N009/Artic045.htm>. Acceso en: 20 december 2006.

NEWELL, P.; KNIGHT, B. Basketball according to knight and newell. Seymour: GraaessleMercer, 1986.

PLATANOU, T. Analysis of the extra man in water polo: A comparison between winning and losing teams and players of different playing position. Journal of Human Movement Studies, Edinburgh, v. 46, p. 205-211, mar. 2004.

RAMPININI, E.; et al. Factors influencing physiological responses to small-sided soccer games. Journal of Sports Sciences, London, v. 25, n. 6, p. 659-666, may 2007.

SAMPAIO, J. Análise de jogo em basquetebol: Estudos e perspectivas. In: TAVARES, F; JANEIRA, M.; GRAÇA, A.; PINTO, D; BRANDÃO, E. Tendências Actuais da Investigação em Basquetebol. Porto: FCDEF-UP, 200I. p. 16-30.

SANDERS, R. H. Analysis of the eggbeater kick used to maintain height in water polo. Journal of Applied Biomechanics, Champaign, v. 15, n. 3, p. 284-291, july 1999a.

SANDERS, R. H. A model of kinematic variables determining height achieved in water polo boosts. Journal of Applied Biomechanics, Champaign, v. I5, n. 3, p. 270-283, july $1999 \mathrm{~b}$.

SMITH, H. K. Applied physiology of water polo. Sports Medicine, v. 26, n. 5, 317-33।, sept. 1998.

SMITH, H. K. Penalty shot importance, success and game context in international water polo. Journal of Science and Medicine in Sport, Mitchell, v. 7, n. 2, p. 221-225, mar. 2004.

STEEL, K. A.; ADAMS, R. D.; CANNING, C. G. Identifying swimmers as water-polo or swim team-mates from visual displays of less than one second. Journal of Sports Sciences, London, v. 25, n. II, p. |25।-1258, dec. 2007.

TSEKOURAS, Y. E. et al. The anthropometrical and physiological characteristics of elite water polo players. European Journal of Applied Physiology, Leeds, v. 95, n. I, p. 35-4I, jan. 2005. 
Recebido: 27 fev. 201 I

Aprovado: 4 set. 2011

Endereço para correspondência:

Francisco Manuel Argudo Iturriaga

Universidad Autónoma de Madrid

Departamento de Educación Física,

Deporte y Motricidad Humana

Avda. Francisco Tomás y Valiente, 3

Campus de Cantoblanco 28049 (Madrid)

España 